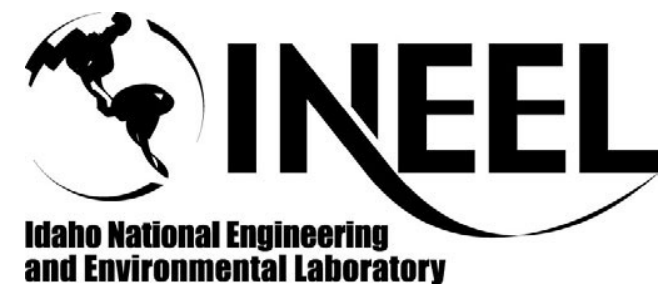

\author{
INEEL/CON-04-02325 \\ PREPRINT
}

\title{
Implementation Of A Hybrid Controller For Ventilation Control Using Soft Computing
}

\author{
Craig G. Rieger \\ D. Subbaram Naidu
}

June 8-10, 2005

\section{American Control Conference}

This is a preprint of a paper intended for publication in a journal or proceedings. Since changes may be made before publication, this preprint should not be cited or reproduced without permission of the author. This document was prepared as an account of work sponsored by an agency of the United States Government. Neither the United States Government nor any agency thereof, or any of their employees, makes any warranty, expressed or implied, or assumes any legal liability or responsibility for any third party's use, or the results of such use, of any information, apparatus, product or process disclosed in this report, or represents that its use by such third party would not infringe privately owned rights. The views expressed in this paper are not necessarily those of the U.S. Government or the sponsoring agency. 


\title{
Implementation of a Hybrid Controller for Ventilation Control Using Soft Computing
}

\author{
Craig G. Rieger and D. Subbaram Naidu Fellow, IEEE
}

\begin{abstract}
Many industrial facilities utilize pressure control gradients to prevent migration of hazardous species from containment areas to occupied zones, often using Proportional-Integral-Derivative (PID) control systems. When operators rebalance the facility, variation from the desired gradients can occur and the operating conditions can change enough that the PID parameters are no longer adequate to maintain a stable system. As the goal of the ventilation control system is to optimize the pressure gradients and associated flows for the facility, Linear Quadratic Tracking (LQT) is a method that provides a time-based approach to guiding facility interactions. However, LQT methods are susceptible to modeling and measurement errors, and therefore the additional use of soft computing methods is proposed for implementation to account for these errors and nonlinearities.
\end{abstract}

\section{INTRODUCTION}

$\mathrm{T}$ HE control of pressure gradients in industrial facilities, such as those found in the Department of Energy (DOE) complex, are key to preventing the migration of hazardous species from containment areas to normally occupied areas. When hatches or doors are opened to access these areas, in some cases for an extended period, the ventilation control system is expected to respond promptly to maintain the required pressure gradients. When the disturbance is maintained for extended periods, operator involvement is often required to rebalance a large portion of the facility to achieve the required balance. Since these control systems often use individual Proportional-IntegralDerivative (PID) or PI controllers, there is no consideration given to the obvious interactions that occur across the facility. To account for these interactions, a control method must provide an optimal solution to the model of a Heating, Ventilation and Air Conditioning (HVAC) plant. The LQT method provides such a solution to optimally track a timebased reference, given that the reference is known and that the model is well known [1], [2]. As the ventilation profiles and pressure gradients through an industrial facility can be

Manuscript received September 15, 2004. Work supported by the U.S. Department of Energy under DOE/NE Idaho Operations Office Contract DE-AC07-99ID13727.

C. G. Rieger is with the Idaho National Laboratory, Idaho Falls, ID, 83415 USA (phone: 208-526-4136; e-mail: Craig.Rieger@inl.gov).

D. S. Naidu is with Idaho State University, Pocatello, ID 83209 USA (phone: 208-282-2307; e-mail: naiduds@isu.edu) quite complex, the presence of modeling and measurement errors must be considered in the final control method.

Soft computing methods provide an avenue to incorporate variations in the model compared to plant operation and the ability to closely model nonlinear situations [3]-[6]. With the LQT method, simulation techniques are available that allow the simple incorporation of any plant model in state space form [7]. However, the implementation of a controller would normally involve the storing of data in the form of a function or lookup table. If multiple tracking references are desired for an individual controller, multiple functions or lookup tables would be required that are activated based on conditions. With a neural network, the data for multiple tracking references can be captured through initial training. With the addition of an integral controller at the local control variable, steadystate offset can be achieved and corrected for variations between the model and plant operations. This design is beneficial in that the model for the LQT can remain simple while still providing an optimized path for the controller to follow.

Soft computing methods also provide for consideration of operator experience. When a ventilation system requires rebalance by an operator, the most experienced will provide the smoothest transition of the plant. As the condition of the plant that mandates the rebalance can change, i.e., doors or hatches that are maintained open in the building can vary, a rule base formed from operator experience is key to controller implementation. This experience can be captured in a fuzzy logic predictor of the most effective tracking references to implement in desired areas of the plant, depending on desired setpoint.

The resulting hybrid controller uses a linear control method, the LQT, and nonlinear methods, neural network and fuzzy logic, to provide an optimized tracking of an HVAC plant as depicted in the flow diagram in Figure 1.

\section{HYBRID CONTROLLER DESIGN}

The block diagram in Fig. 2 represents the hybrid controller that is considered for controlling an HVAC system. The design involves the development of a global LQT controller, training of a neural network with the LQT 


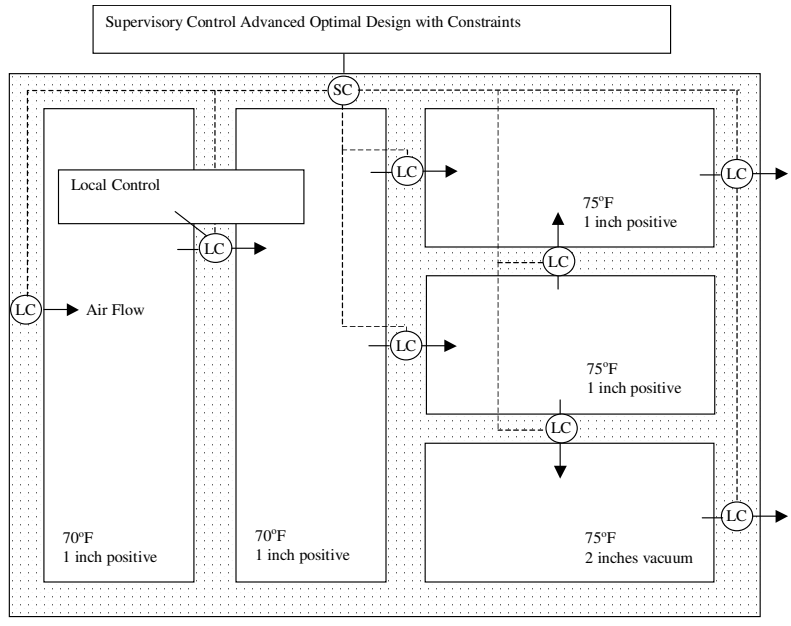

Fig. 1. Supervisory HVAC Plant Control

data, development of global fuzzy-based track reference, and final combination with an integrator for local control.

\section{A. $L Q T$}

The development of a LQT controller for maintaining pressure gradients starts with the development of a model for the HVAC system. Consider the 3-cell ventilation situation depicted in Fig. 1. A simple state-space model for pressure and flow was developed using a differential form of the ideal gas law [7]-[9].

Assuming little change in temperature, then linearizing for each cell:

$\dot{\mathrm{x}}(\mathrm{t})=\mathrm{K}_{\mathrm{a}} \mathrm{x}(\mathrm{t})+\mathrm{K}_{\mathrm{b}} \mathrm{u}(\mathrm{t})$

where:

$x(t)$ - state vector, pressure,

$u(t)$ - input vector, flow,

$K_{a}, K_{b}$ - constants, and

For the overall 3-cell system:

$$
\dot{\mathrm{x}}(\mathrm{t})=\mathrm{K}_{\mathrm{taX}}(\mathrm{t})+\mathrm{K}_{\mathrm{tb}} \mathrm{U}_{\mathrm{o}}(\mathrm{t})
$$

where:

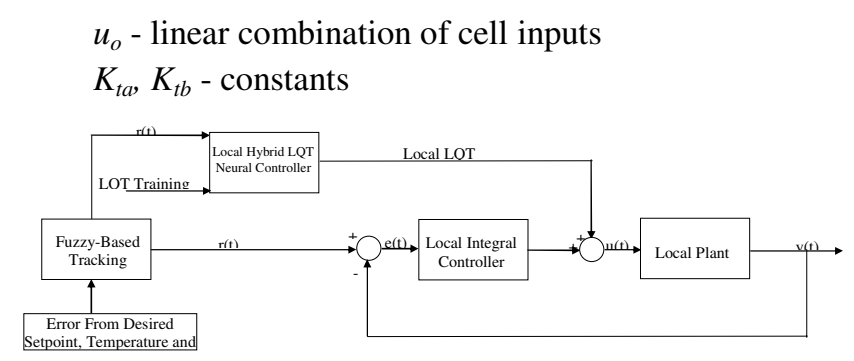

Fig. 2. HVAC Hybrid Controller Design
Although only pressure has been mentioned as it is the primary state of concern in this paper, temperature must also be considered in the final hybrid controller. Wide swings in the temperature can cause discomfort and unacceptable working conditions for those that enter containment areas for work, especially considering the fact that those persons are often wearing personal protective equipment (PPE). While many works have developed models for temperature control, the application of constrains for temperature in this work will be included in the fuzzy-based tracking method [2], [10], [11].

The final state-space model for the ventilation system appears as follows:

$$
\begin{aligned}
{\left[\begin{array}{l}
\dot{x} 1 \\
\dot{x} 2 \\
\dot{x} 3 \\
\dot{x} 4
\end{array}\right]=\left[\begin{array}{cccc}
-19280 & 0 & 0 & 0 \\
0 & -19280 & 0 & 0 \\
0 & 0 & -19280 & 0 \\
0 & 0 & 0 & -57840
\end{array}\right]\left[\begin{array}{l}
x 1 \\
x 2 \\
x 3 \\
x 4
\end{array}\right]+\left[\begin{array}{ccc}
5427 & 0 & 0 \\
0 & 5427 & 0 \\
0 & 0 & 5427 \\
5427 & 5427 & 5427
\end{array}\right]\left[\begin{array}{l}
u 1 \\
u 2 \\
u 3
\end{array}\right] } \\
{\left[\begin{array}{l}
y 1 \\
y 2 \\
y 3 \\
y 4
\end{array}\right]=\left[\begin{array}{llll}
1 & 0 & 0 & 0 \\
0 & 1 & 0 & 0 \\
0 & 0 & 1 & 0 \\
0 & 0 & 0 & 1
\end{array}\right]\left[\begin{array}{l}
x 1 \\
x 2 \\
x 3 \\
x 4
\end{array}\right] }
\end{aligned}
$$

The resulting plant model can be used to develop an LQT controller using recent results by the authors [7]. The normal state-space representation of a system is provided below

$$
\begin{aligned}
& \dot{x}(t)=A x(t)+B u(t), \\
& y(t)=C x(t) .
\end{aligned}
$$

where $\mathrm{A}, \mathrm{B}$ and $\mathrm{C}$ are of appropriate dimensions, and $y(t)$ is the output vector.

The objective is to minimize the error $(e)$ between a time-varying tracking reference $(z)$ and the output $(y)$ [1], [12], [13]. The error vector is therefore defined as

$$
e(t)=z(t)-y(t)=z(t)-C x(t) .
$$

To minimize the tracking error and the expenditure of control effort, a performance index is chosen as

$$
J=\frac{1}{2} e^{\prime}(t f) F e(t f)+\frac{1}{2} \int_{t 0}^{t f}\left[e^{\prime}(t) Q e(t)+u^{\prime}(t) R u(t)\right] d t .
$$

The LQT Hamiltonian canonical representation takes the form

$$
\left[\begin{array}{l}
\dot{x} *(t) \\
\dot{\lambda} *(t)
\end{array}\right]=\left[\begin{array}{cc}
A & -B R^{-1} B^{\prime} \\
-C^{\prime} Q C & -A^{\prime}
\end{array}\right]\left[\begin{array}{l}
x *(t) \\
\lambda^{*}(t)
\end{array}\right]+\left[\begin{array}{c}
0 \\
C^{\prime} Q
\end{array}\right] z(t)
$$


and the LQT Riccati equation as

$$
\dot{P}(t)=-P(t) A-A^{\prime} P(t)+P(t) B R^{-1} B^{\prime} P(t)-C^{\prime} Q C
$$

with boundary condition at final time $t_{f}: P\left(t_{f}\right)=C^{\prime}\left(t_{f}\right) F C\left(t_{f}\right)$, and the LQT vector differential equation

$$
\dot{g}(t)=\left[P(t) B R^{-1} B^{\prime}-A^{\prime}\right] g(t)-C^{\prime} Q z(t)
$$

with boundary condition at final time $t_{f}: g\left(t_{f}\right)=C^{\prime}\left(t_{f}\right) F z\left(t_{f}\right)$.

Solutions of these equations for the Riccati coefficient $(P)$ and vector $(g)$ is dependent on design parameters $Q, R$ and $F$ defined in the performance index. The resulting optimal closed loop LQT controller (u) takes the form

$$
u(t)=-R^{-1} B^{\prime} P(t) x(t)+R^{-1} B^{\prime} g(t) .
$$

Using the analytical solution to the matrix differential Riccati Equation and extending it to the LQT problems provides a technique for providing the controller and outputs for a given time period [7]. This technique is implemented in Matlab ${ }^{\circledR}$, but is generally applicable.

\section{B. Neural Network Based LQT}

The output and controller data can be used as training data set for a predictive controller. The resulting controller provides a useful and flexible alternative to developing a lookup table for the LQT data, which can accept different reference inputs and provide an LQT output. However, it must be noted that delays in the neural network implementation make the resulting controller suboptimal [14].

With the cause-effect relationship between the inputs and outputs allows them to be paired, the neural network will be implemented as local controllers [7], [15]. The neural network technique used is based on the receding horizon technique. The neural network model provides a control output over a specified time horizon, and is built into the Matlab $^{\circledR}$ control toolbox. The predictions are used by a numerical optimization program to determine the control signal that minimizes a given performance criterion shown below:

$$
\sum_{j=N_{1}}^{N_{2}}\left(y_{r}(t+j)-y_{m}(t+j)\right)^{2}+\rho \sum_{j=1}^{N u}\left(u^{\prime}(t+j-1)-u^{\prime}(t+j-2)\right)
$$

where $N_{l}, N_{2}$, and $N_{u}$ define the horizons over which the tracking error and the control increments are evaluated. The $u$ ' variable is the tentative control signal, $\mathrm{y}_{\mathrm{r}}$ is the desired response and $\mathrm{y}_{\mathrm{m}}$ is the network model response. The $\rho$ value determines the contribution that the sum of the squares of the control increments has on the performance index.
The neural network used is composed of three delayed inputs, three delayed outputs and three hidden layers. Figures 3 and 4 depict the input and output data of the LQT and LQT-trained neural network without disturbances. Note that the plant outputs are smooth at the beginning and endpoints, resulting from the neural network optimization scheme smoothing LQT controller response.

\section{Fuzzy Tracking Reference}

Normally the reference trajectories that will be implemented for each control variable would be dependent solely on the layout of the HVAC flow balance. This method requires a significant modeling effort to account for plant nonlinearities that occur during many potential disturbance conditions. A much easier approach is to embed knowledge of plant operations into a fuzzy controller, which will also ensure temperature control is factored in to the overall control scheme. This is crucial when plants have an upstream air-handling unit (AHU),

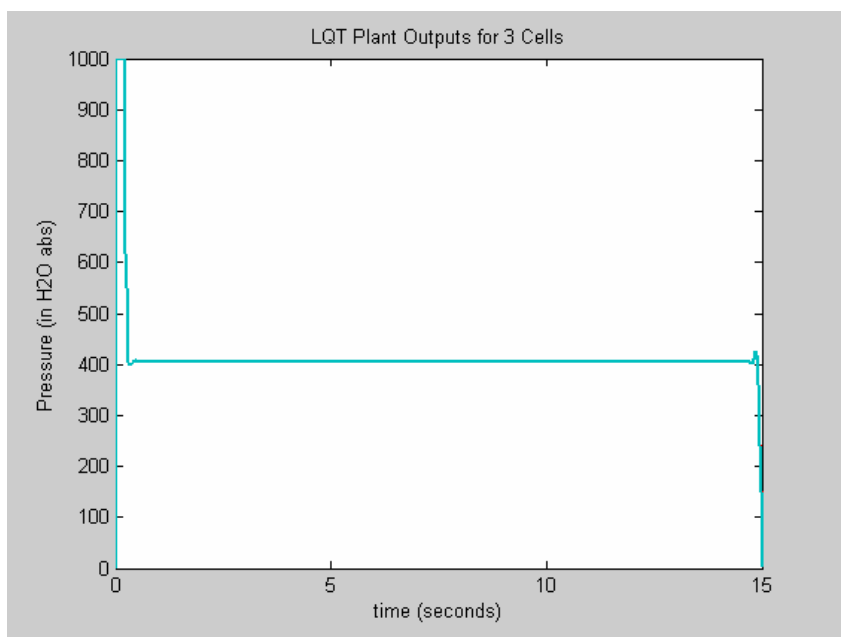

Fig. 3. 3-cell Plant Outputs with LQT Controller

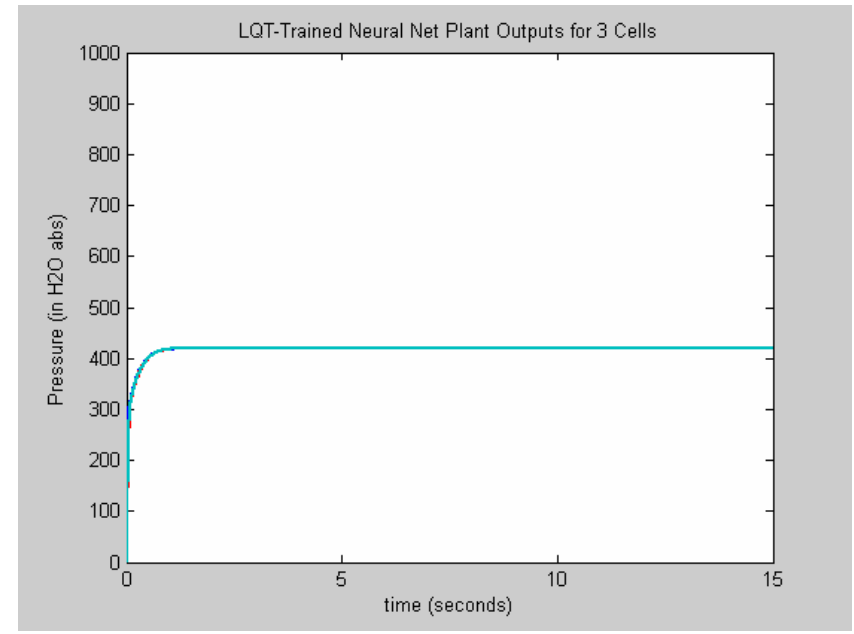

Fig. 4. 3-cell Plant Outputs with Neural Net Controller 
which provides a single setpoint for the discharge temperature that feeds all the cells. For ease of implementation in Matlab ${ }^{\circledR}$, a Mamdani scheme is chosen.

The Mamdani fuzzy logic scheme used has three outputs and six inputs, which includes for each cell, the error between the pressure and the desired setpoint and the same for temperature [16]. Trapezoidal membership functions (MF) were used, with three MF on each of the three pressure error inputs, five $\mathrm{MF}$ on the temperature error inputs and five MF on the three outputs. This arrangement allows for creating a rule set that grades the relative importance of pressure over temperature control, as any shift in pressure requires correction but small changes in temperature do not.

The rule set is designed not only to place higher priority on pressure error, but to also place priority on Cell 1 pressure gradients over that of the other cells. In this way

Table 1. Fuzzy Rule Base

\begin{tabular}{|c|c|c|c|c|c|c|c|c|}
\hline $\begin{array}{c}\text { Cell1 } \\
\text { Pres. } \\
\text { Err } \\
\end{array}$ & $\begin{array}{c}\text { Cell2 } \\
\text { Pres. } \\
\text { Err } \\
\end{array}$ & $\begin{array}{c}\text { Cell3 } \\
\text { Pres. } \\
\text { Err } \\
\end{array}$ & $\begin{array}{c}\text { Cell1 } \\
\text { Temp. } \\
\text { Err } \\
\end{array}$ & $\begin{array}{c}\text { Cell1 } \\
\text { Temp. } \\
\text { Err } \\
\end{array}$ & $\begin{array}{c}\text { Cell1 } \\
\text { Temp. } \\
\text { Err } \\
\end{array}$ & Out1 & Out2 & Out3 \\
\hline Low & N/A & N/A & Ok & N/A & N/A & $\sim \mathrm{High}$ & N/A & N/A \\
\hline Ok & N/A & N/A & $\sim$ Low & N/A & N/A & Ok & N/A & N/A \\
\hline High & N/A & N/A & Low & N/A & N/A & $\sim$ High & N/A & N/A \\
\hline High & N/A & N/A & Ok & N/A & N/A & $\sim$ Low & N/A & N/A \\
\hline Ok & N/A & N/A & $\sim$ High & N/A & N/A & Ok & N/A & N/A \\
\hline Ok & N/A & N/A & High & N/A & N/A & $\sim$ Low & N/A & N/A \\
\hline Low & N/A & N/A & Low & N/A & N/A & High & N/A & N/A \\
\hline High & N/A & N/A & High & N/A & N/A & Low & N/A & N/A \\
\hline N/A & Low & N/A & N/A & Ok & N/A & N/A & $\sim$ High & N/A \\
\hline N/A & Ok & N/A & N/A & $\sim$ Low & N/A & N/A & Ok & N/A \\
\hline N/A & Ok & N/A & N/A & Low & N/A & N/A & $\sim$ High & N/A \\
\hline N/A & High & N/A & N/A & Ok & N/A & N/A & $\sim$ Low & N/A \\
\hline N/A & Ok & N/A & N/A & $\sim$ High & N/A & N/A & Ok & N/A \\
\hline N/A & Ok & N/A & N/A & High & N/A & N/A & $\sim$ Low & N/A \\
\hline N/A & Low & N/A & N/A & Low & N/A & N/A & High & N/A \\
\hline N/A & High & N/A & N/A & High & N/A & N/A & Low & N/A \\
\hline N/A & N/A & Low & N/A & N/A & Ok & N/A & N/A & $\sim$ High \\
\hline N/A & N/A & Ok & N/A & N/A & $\sim$ Low & N/A & N/A & Ok \\
\hline N/A & N/A & Ok & N/A & N/A & Low & N/A & N/A & $\sim \mathrm{High}$ \\
\hline N/A & N/A & High & N/A & N/A & Ok & N/A & N/A & $\sim$ Low \\
\hline N/A & N/A & Ok & N/A & N/A & $\sim$ High & N/A & N/A & Ok \\
\hline N/A & N/A & Ok & N/A & N/A & High & N/A & N/A & $\sim$ Low \\
\hline N/A & N/A & Low & N/A & N/A & Low & N/A & N/A & High \\
\hline N/A & N/A & High & N/A & N/A & High & N/A & N/A & Low \\
\hline Low & Low & Ok & N/A & N/A & N/A & High & $\sim$ High & Ok \\
\hline Low & Ok & Low & N/A & N/A & N/A & High & Ok & $\sim$ High \\
\hline High & High & Ok & N/A & N/A & N/A & Low & $\sim$ Low & Ok \\
\hline High & Ok & High & N/A & N/A & N/A & Low & Ok & $\sim$ Low \\
\hline
\end{tabular}

the control response to disturbances that affect each cell, such as the incoming pressure, would affect Cells 2 and 3 more than Cell 1. Table 1 is a listing of the 28 rules used.

\section{Integral Controller}

An integral controller is placed on each cell to provide a zero steady-state offset. The contribution of this controller and the local neural net controller are added. The integral constant used is the same as that used with a PI implementation of the same plant, which will be used as a comparison of results in the next section.

\section{RESULTS AND DISCUSSION}

The results that follow are provided by a Matlab ${ }^{\circledR}$ representation and simulation of the hybrid controller depicted in Figure 2. LQT controller data and training of the neural network occurred before the simulation of the hybrid controller. Step disturbances are injected into the system, which include two flow step disturbances in Cell 1, and a temperature step disturbance, also in Cell 1. The flow disturbances are less than $1 \%$ of the absolute flow and the temperature disturbance is a $15 \%$ disturbance. These were selected based on the type of disturbances expected in a plant, albeit the flow disturbances correlate better to a step than the temperature, which would more often be expected to be a ramp when a heat-generating process goes into operation. However, a step response provides a more effective tool at determining the effectiveness of the controller. For this simulation it is assumed that the supply air is hotter than the cell air, such as in winter conditions.

Figure 5 depicts the fuzzy tracker output based on the startup differential and disturbances. Due to the size of the error, the pressure gradient on the initial startup and the temperature disturbance cause a transition in the tracking output of the fuzzy tracker. The initial transition affects all cells, but the latter affects only Cell 1 in line with the disturbance. Between 4 and 12 seconds the fuzzy tracker does not give a response, which is desirable and prevents the neural controller from tracking unnecessary changes

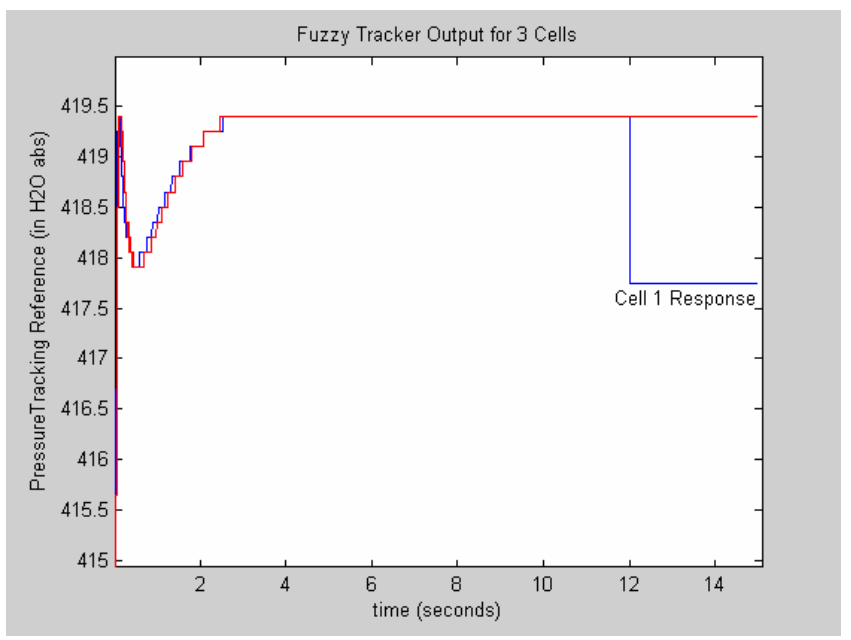

Fig. 5. 3-Cell Fuzzy Tracker Output 
and minimizes control energy.

For comparison purposes, the same plant was simulated with PI controllers. In addition, the same flow disturbances were created. The simple PI controller system is designed to look at pressure only on a local level, and therefore this scheme lacks the capability of the hybrid controller to consider temperature. Figures 6 and 7 depict the controller outputs to the 3-cell plant. It is worth noting that the hybrid controller provided improvements over simple PI control, leveling off quicker and providing appropriate corrections for temperature variations. In response to the fuzzy rule base, minor disturbances do not impact the tracking reference. This is an appropriate application of the integral controller, which ensures a zero steady state offset with minimal control energy. Similar offsets would be expected for modeling and measurement errors, and the primary reason for its inclusion in the hybrid controller design.

The pressure response of the plant is provided in Figure 8. As the fuzzy tracker rule set favors Cell 1 pressure and therefore has a more aggressive response to out-ofspecification conditions, it is noted that the initial response peak for Cell 1 is somewhat reduced compared to the peaks

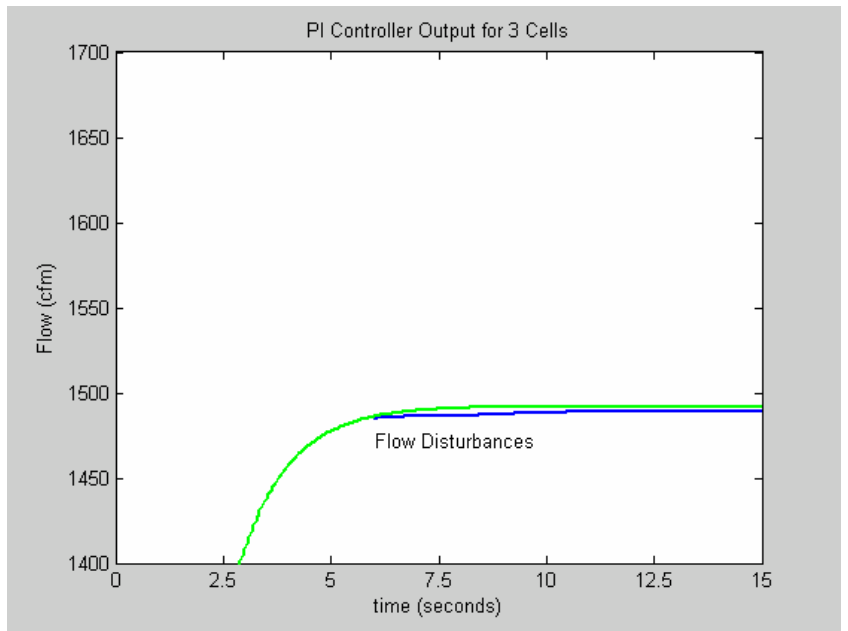

Fig. 6. 3-Cell Simple PI Controller Output

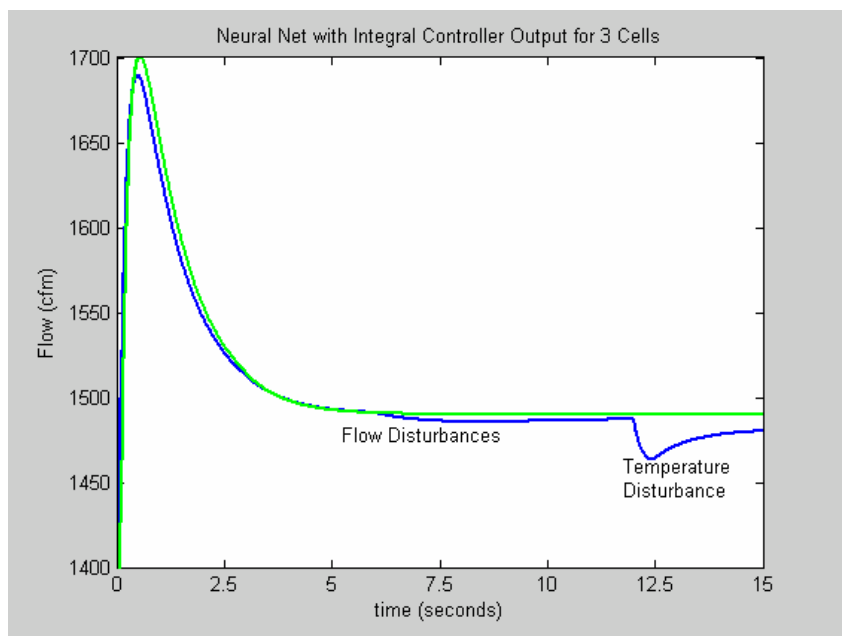

Fig. 7. 3-Cell Neural Net with Integral Controller for the other two cells. The flow disturbances into Cell 1, both an increase and a decrease, create an expected increase in pressure, then decrease. The temperature increase in Cell 1 causes a direct response from the fuzzy tracker, as mentioned earlier, causing an immediate decrease in flow of hot air to the room resulting in cooling.

\section{CONCLUSIONS}

While energy efficiency are key to many advanced control designs in the area of HVAC, industrial facilities such as those in the DOE complex focus on pressure controls to prevent migration of hazardous substances. However, temperature controls must also be considered in the design, as personnel with PPE must enter these areas to perform maintenance tasks. Simple PID or PI designs do not consider the performance of the plant as a whole, or provide a global control. However, LQT controls can provide outstanding control for an entire plant. Implemented using a neural network and fuzzy inference system providing a tracking reference, the data provided have demonstrated that a hybrid controller can be implemented which focuses on the need to optimize global pressure control while still providing consideration of temperature effects. Proper application of a rule base can ensure that the fuzzy tracker prioritizes the control responses to key control variables, while minimizing control energy. Inclusion of an integral controller can offset small disturbances and modeling errors in the LQT design, reducing the overall effort required by the hybrid controller.

Additional research is being performed to implement the principles described in this paper on a small HVAC experimental system. Testing will confirm the ease of application of the methodology described and provide a basis for establishing the control design parameters, such as the integral gain and neural network weights, in an operational system with associated nonlinearities.

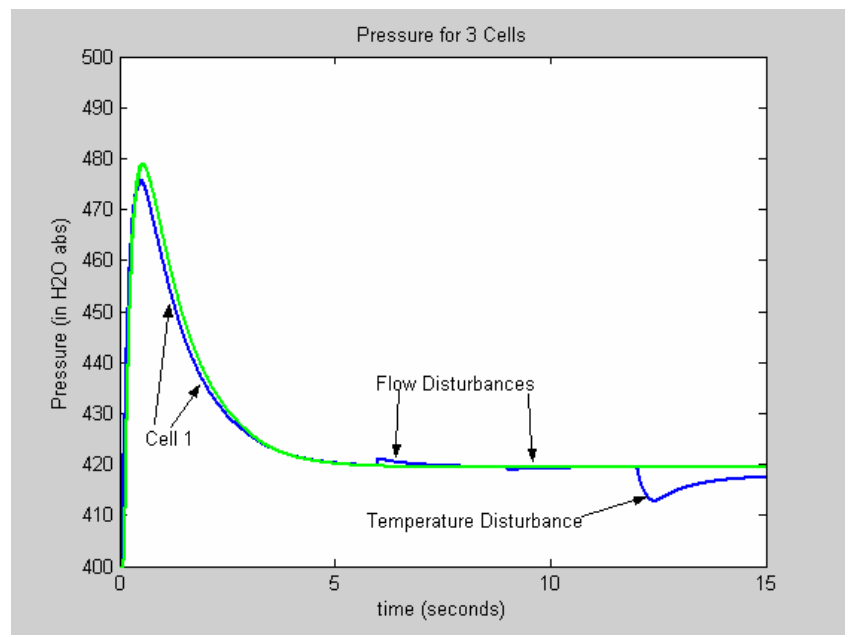

Fig. 8. Pressure in the 3 Cells 


\section{REFERENCES}

[1] D. S. Naidu, Optimal Control Systems, CRC Press, Boca Raton, 2003.

[2] M. Zaheer-uddin and R. V. Patel, "Optimal Tracking Control of Multi-Zone Indoor Environmental Spaces," Transactions of the ASME, Journal of Dynamic Systems, Measurement, and Control, “ 1995, 117, pp. 292-303.

[3] S. J. Hepworth and A. L. Dexter, "Neural Control of Non-linear HVAC Plant," Proceedings of the IEEE Conference on Control Applications, 1994, 3, 1849-1854.

[4] B. Arguello-Srrano and M. Vlez-Reyes, "Nonlinear Control of a Heating, Ventilating, and Air Conditioning System with Thermal Load Estimation," IEEE Transactions on Control Systems Technology, 1999, 7, 56-63.

[5] P. S. Curtiss, G. Shavit and J. F. Kreider, "Neural Networks Applied to Buildings-A Tutorial and Case Studies in Prediction and Adaptive Control," ASHRAE Transactions: Symposia, 1996, 102, pp. 1141-1166.

[6] A. Zilouchian and M. Jamshidi, Intelligent Control Systems Use Soft Computing Technologies, CRC Press, Boca Raton, 2001.

[7] C. G. Rieger and D. S. Naidu, "New Techniques for Implementing Linear Quadratic Methods with Aerospace and Other Industrial Control Applications," Proceedings of the Sixth IASTED International Conference on Intelligent Systems and Control, Honolulu, Hawaii, 2004, pp. 388-393.

[8] C. G. Rieger, Advanced Control Strategies for HVAC Systems in Critical Building Structures, Internal Report, Idaho State University, 2002.

[9] ASHRAE Fundamentals Handbook CD, 2001.

[10] C. P. Underwood, HVAC Control Systems: Modeling, Analysis and Design, Routledge, New York, 1999.

[11] C. P. Underwood, "Robust control of HVAC," Proceedings of the Chartered Institution of Building Services Engineers, 2000, 21, pp. 63-71.

[12] M. Athans and P. Falb, Optimal Control, McGraw-Hill, New York, 1966.

[13] B. D. O. Anderson and John B. Moore, Optimal Control Linear Quadratic Methods, Prentice Hall, Englewood Cliffs, 1990.

[14] K. L. Moore, D. S. Naidu and M. Siddaiah, "A Real-time Adaptive Linear Quadratic Regulator Using Neural Networks," European Control Conference (ECC), Groningen, The Netherlands, 1993.

[15] T. E. Marlin, "Process Control: Designing Processes and Control Systems for Dynamic Performance," McGraw-Hill, New York, 1995.

[16] J. Jantzen, “Tuning of Fuzzy PID Controllers,” Technical University of Denmark, Technical Report \#98-H 871, 1998. 